\title{
MOVIMIENTOS SOCIALES, NUEVA RAZÓN DE ESTADO Y LA ESTIGMATIZACIÓN DE LA PROTESTA SOCIAL EN COLOMBIA*
}

DOI: http://dx.doi.org/10.17981/juridcuc.11.1.2015.14

Recibido: 11 de Mayo de 2015 / Revisado: 27 de Julio de 2015 / Aceptado: 28 de Julio de 2015

\author{
Miguel Antonio Morón Campos** \\ Fundación Universitaria Tecnológico COMFENALCO - Sede Cartagena \\ Puede citar el presente artículo así: / To reference this article: \\ Morón, M. (2015). Movimientos Sociales, Nueva Razón de Estado y la Estigmatización de la Protesta Social \\ en Colombia. Jurídicas CUC, 11(1), 311-326. doi: http://dx.doi.org/10.17981/juridcuc.11.1.2015.14
}

\section{Resumen}

El objeto de este trabajo consiste en explicar las dinámicas sociales y políticas que supone el ejercicio de libertad de reunión en un Estado democrático. Bajo una perspectiva explicativa se desarrollarán una serie de hipótesis en las que se establecen los vínculos entre movimientos sociales, neoliberalismo y la criminalización de la protesta social, poniendo en evidencia como las dinámicas políticas soportadas en la desterritorialización de la economía, la privatización de garantías sociales y políticas, así como el endurecimiento de políticas legales que reducen libertades sociales, desdibujan los propósitos colectivos de los movimientos sociales en Colombia.

\section{Palabras clave:}

Movimientos sociales, razón de Estado, protesta social, acción colectiva, gubernamentalidad

\footnotetext{
" Este artículo de investigación es resultado del proyecto "Políticas Públicas, movimientos sociales y la criminalización de la protesta social en Colombia: 1978-2015" en la cual el autor figura como Investigador Principal. Investigación desarrollada al interior el grupo de investigación Sociales y Jurídicas de la Fundación Universitaria tecnológico Comfenalco-Cartagena.

** Abogado egresado de la Universidad de Cartagena. Miembro del grupo de investigaciones Sociales y Jurídicas de la Fundación Universitaria Tecnológico Comfenalco-Cartagena. Director del semillero Castigo y Sociedad. Estudiante de la Maestría Conflicto Social y Construcción de paz de la Universidad de Cartagena. Correo Electrónico: mmoron@tecnocomfenalco.edu.co
} 


\section{SOCIAL MOVEMENTS, NATIONAL INTEREST AND SOCIAL PROTEST STIGMA IN COLOMBIA}

\section{Abstract}

The purpose of this paper is to explain the social and political dynamics involved the exercise of freedom of assembly in a democratic State. Under an explanatory perspective, a number of scenarios in which the links between social movements, neoliberalism and the criminalization of social protest are established showing how the political dynamics supported in the deterritorialization of the economy, the privatization of social and political guarantees as well as the tightening of legal policies that reduce social freedoms, blur the collective purposes of social movements in Colombia.

\section{Keywords:}

Social Movements, National Interest, Social Protest, Collective Action, Governmentality. 
MOVIMIENTOS SOCIALES, NUEVA RAZÓN DE ESTADO Y LA

\section{INTRODUCCIÓN}

Las nuevas formas en las que se configuran las relaciones sociales se articulan a dinámicas y prácticas discursivas con las que se comprende la política y la economía desde el Estado. Sin embargo, el leviatán obedece a una racionalidad diferente y su imagen ya no es acción en la que todo lo coacciona y lo ejerce para reafirmar su autoridad, ahora se proyecta como efecto de las relaciones de poder que le preceden (Foucault, 2001, p. 219): toda una multiplicidad de relaciones microfísicas que convergen en y desde el territorio.

Esto es en lo que la analitica del poder se ha denominado como procesos de estatalización o gubernamentalización del Estado, en la que éste ya no es asimilado como un "monstruo frío, como una institución autónoma y dotada de una racionalidad propia, sino como el espacio inestable por donde se cruzan diferentes tecnologías de gobierno" (Foucault, 2001, p. 220). El Estado, en ese sentido, solo es posible a partir de una praxis de los actores que son externos a él.

Bajo estos presupuestos, el objetivo de este avance de investigación consiste en explicar los alcances analíticos de la categoría movimientos sociales a partir de sus efectos políticos en contextos democráticos. En ese sentido, a partir de tres momentos diferenciables, se abordará de manera reflexiva, por un lado, las condiciones de posibilidad de la acción colectiva en contextos neoliberales, y por otro, los efectos de políticas institucionales a delimitar la libertad de reunión y el derecho de protesta en contextos democráticos. Para ello primero se describirán algunos marcos explicativos con los que se comprenden los Nuevos Movimientos Sociales y la acción colectiva; seguidamente se analizarán las dinámicas socioespaciales a partir de la categoría gubernamentalidad; el tercer momento abordará cómo esas dinámicas socio-espaciales se consolidan a partir de políticas represivas que han criminalizado el derecho de protesta en Colombia; y por último, se plantearán una serie de conclusiones reflexivas sobre los alcances legales y políticos de los movimientos sociales en el territorio nacional. 


\section{METODOLOGÍA}

Esta investigación de corte cualitativo tiene como propósito, desde una perspectiva socio-histórica, una reconstrucción analítica en torno a la emergencia de los movimientos sociales en Colombia a partir de las diferentes dinámicas glocales que la acción colectiva supone. Ello implica abordar el objeto de estudio a partir de un análisis que arroje las cualidades del mismo a través de diferentes momentos en clave con la comprensión de la realidad social como proceso de construcción permanente (Ragin, 2007, p. 15).

Lo anterior, se traduce en la necesidad de adoptar una postura metodológica de carácter dialógico y documental que permita acceder a los diferentes marcos explicativos y descriptivos de los movimientos sociales a partir de un estudio sistemático y coherente, integrado por los discursos académicos que han tratado de explicar esos fenómenos y por el impacto social que ha generado las diferentes intervenciones institucionales en los movimientos sociales.

$\mathrm{Al}$ ser entonces, una investigación cualitativa, intentamos captar el punto de vista desde el cual los movimientos sociales participan en la configuración de la garantía constitucional del derecho de reunión y la criminalización de la protesta social, principalmente aquellos que de manera directa contribuyeron a configurar esa realidad en el marco de los procesos de reconocimiento de las luchas sociales por parte de las instituciones públicas (Ragin, 2007, p. 23).

Esta investigación contribuirá a la comprensión analítica y estratégica de las condiciones de posibilidad de los movimientos sociales en Colombia a partir del uso de la acción colectiva, generando un acercamiento hacia las garantías sociales y el reconocimiento del derecho de protesta en las dinámicas sociales locales. Al ser el objeto del mismo los alcances teóricos que han construido buena parte de las luchas sociales en Colombia, los resultados de esta investigación permitirán la directa participación de colectivos al interior de los procesos de autoconciencia y construcción de un nuevo porvenir, en el cual la experiencia de la criminalización de la protesta haya podido ser asimilada y superada. 


\section{Nuevos Movimientos Sociales: aproximación conceptual}

La categoría Nuevos Movimientos Sociales (NMS) emerge para explicar ciertas formas de acción colectiva surgidas en la segunda mitad de los años sesenta del siglo XX, las cuales hacían una ruptura con los marcos explicativos que abordaban temas relacionados con la acción colectiva y la acción social (Delgado, 2005, p .32). Bajo esta etiqueta se pretenderá describir y explicar el conjunto de formas de acciones colectivas que superan una lectura del campo social a partir de la división de clases sociales con los que venía abordándose desde la Revolución Industrial hasta poco después de la Segunda Guerra Mundial (Melucci, 1996, p. 122).

En ese sentido, esta categoría analítica tiene como motivación comprender los límites de ciertas tradiciones teóricas que elaboran sus análisis a partir de una perspectiva interaccionista, la cual se sustenta en los siguientes presupuestos (Neveu, 2000, p. 118):

1. Los movimientos sociales a partir de un proceso continuo y dinámico de construcción social, el cual se explica a partir de las significaciones del contexto y las condiciones culturales por las cuales emerge atendiendo a los signos con que se representa socialmente.

2. Los procesos de rotulación discursiva en torno a los movimientos se construyen a partir de los problemas por los cuales emerge.

3. Las acciones que se desprenden de los movimientos sociales están cargadas de motivación, lo cual supone que estos pueden redefinir permanentemente los fines con los que se ha creado y reorientarlos a partir de las transformaciones del contexto.

Así, los estudios que han tenido por objeto comprender la emergencia de los NMS guardan relación al explicar que las formas de expresión colectiva que en ellos convergen, están motivadas en problemáticas referentes a la edad, la orientación sexual, la afinidad con sectores excluidos o gremios profesionales afectados por políticas nacionales o transnacionales; dejando en un segundo plano los movimientos que tienen motivaciones ideológicas o partidistas. Los presupuestos meto- 
dológicos con los que se distinguen los NMS atienden a un ejercicio de identificación de los diferentes sujetos que lo conforman, así como las estrategias necesarias que sirven de plataforma para la movilización (Delgado, 2005, p. 44).

Es importante comprender los movimientos sociales como "agentes de significación colectiva que difunden nuevos significados en la sociedad a través de acción colectiva" (Melucci, 1994, p. 120); a partir del análisis desarrollado por Delgado (2005, p. 31), este concepto debe comprenderse a partir de los siguientes puntos:

1. Que los movimientos sociales apelan a la solidaridad entendida como la capacidad de sus miembros para definir y reconocer un sentido del "nosotros", y desde ahí compartir y construir una Identidad Colectiva como producto del proceso de atribución de significado y de las cambiantes situaciones que motivan la Acción Colectiva;

2. Que la movilización explicita un conflicto social en la medida en que los actores perciben una condición problemática o un aspecto de sus vidas no simplemente como una desgracia sino como una injusticia, por lo cual se configura paulatinamente un marco de interpretación compartido desde el que justifican y legitiman su Acción Colectiva, y

3. Que la acción busca romper los límites del sistema en que se produce, por lo cual la capacidad del movimiento para provocar rupturas en las fronteras del sistema de relaciones sociales de poder en el que se desarrolla su acción política, se considera básica para diferenciar los movimientos de otros fenómenos que no tienen la intención de producir cambios en el sistema de normas y de relaciones sociales.

Con estas consideraciones, comprender los NMS significa abordarlos a partir de su motivación por la transformación de las condiciones materiales en las que éstos se asientan, la cual solo es posible en el marco de una dimensión política de la acción colectiva (Delgado, 2005, p. 32), con ello, siguiendo a Melucci, los NMS son proyectados a partir de la interacción, pues las reglas de definición en torno a la deliberación es la que posibilita delimitar sus objetivos, todo ello en un marco explicativo que pueda poner en evidencia las garantías políticas y los límites de sus acciones (Melucci, 1999, p. 36). 


\section{Una nueva razón de Estado: la gubernamentalidad como presupuesto teórico para comprender las dinámicas socio-espaciales}

Las dinámicas que comprenden las relaciones socio-espaciales atraviesan un proceso de transformación en las que el neoliberalismo se autopostula como el mejor de los modelos para el mejoramiento permanente del planeta. Con él se anuncia el nuevo evangelio del desarrollo económico: "[l]as recetas de estabilización monetaria, el ajuste estructural y el estímulo de estrategias de crecimiento económico basadas en la apertura de mercados, el apoyo a exportaciones, la búsqueda de la inversión extranjera y la cohesión social” (Jiménez \& Novoa, 2014, p. 30).

En ese sentido, los protagonistas que le dan forma a esta serie de propuestas enuncian sus palabras desde la economía. Si bien el neoliberalismo acoge problemas tan diversos como contradictorios: eficiencia económica, equidad social, bienestar del medio ambiente, etc., esta pluralidad de problemas supone comprender su horizonte de proyección como difuso. La categoría que supone comprender el neoliberalismo mantiene una diversidad de prácticas sociales, ello permite afirmar que existen "neoliberalismos"; sin embargo, todos ellos podrían guardar ciertas similitudes: "reestructurar las formas de organización de la producción y gestión del trabajo, y reformar las estrategias de dominio y disciplina social" (Jiménez \& Novoa, 2014, p. 29). No obstante, con en esta serie de prácticas no se limita a dominar a otros por la fuerza (practicas perfeccionadas desde el lenguaje del derecho), lo que es particular en este discurso es que pretende dirigir a la población para que sus acciones sean eficaces y mantengan su consentimiento, es decir, la instrumentalización de la libertad de aquellos que son gobernados. Con el neoliberalismo se crean ciertas "condiciones de aceptabilidad en donde los sujetos se experimentan a sí mismos como libres, aunque los objetivos de su conducta sean puestas por otros" (Castro, 2010, p. 12). A este punto, las relaciones entre poder y libertad mantienen un mismo horizonte en el que, a partir del neoliberalismo y la economía capitalista, asciende una nueva razón de Estado.

En las transformaciones de la economía se reproducen los problemas que supone la cuestión espacial pues, a partir de la globalización, 
se trazan nuevos centros de producción económica, des-localizando la materialización de la misma, y con ello, procesos de des-territorialización, los cuales están soportados por los intereses del capital (Jiménez \& Novoa, 2014, p. 31).

Todas estas dinámicas en las cuales transitan y desarraigan los cuerpos se encuentran legitimadas por la Estatalidad y el Derecho. En ese sentido, lo que permitirá el neoliberalismo, en esta nueva dinámica de las relaciones sociales y económicas, será cambiar la ecuación de la función del Estado frente a sus asociados: a éste no le interesan las garantías y el bienestar del individuo, ahora se concentra en las garantías y estabilidad del sistema (Jiménez \& Novoa, 2014, p. 32).

La otra cara que soporta toda esta dinámica de la estatalidad es la globalización, pues ésta, en tanta plataforma para las prácticas económicas, políticas y culturales, es asumida a partir de la expansión permanente de los procesos que en ella se entrelazan. De esta forma, los efectos que en ella convergen suponen un cambio en las dinámicas espacio-temporales de las relaciones sociales (Jiménez \& Novoa, 2014, p. 25).

La globalización es simultáneamente espacial (basada en una continua extensión, configuración y reestructuración de la organización territorial del capitalismo) y temporal (basada en una aceleración continua del circuito medio del capital) (Jiménez \& Novoa, 2014, p. 26).

En ese sentido, las prácticas que convergen en la globalización suponen la ascensión de la economía como fundamento de las relaciones sociales. El capitalismo reproduce permanentemente sus formas a partir de esta plataforma en la que ya no existen fronteras, y con ello, un cambio en la forma como se asimila el mundo. Desde el modelo económico, bajo el estandarte del desarrollo y la innovación, las relaciones sociales pretenden abrazar este tipo de propuestas, suponiendo que con ello se podrá pensar el mejor de los mundos posibles.

A este punto, comprendiendo las relaciones entre economía y razón de Estado, puede comprenderse que el rostro político del discurso que supone los efectos de la globalización es el neoliberalismo. Su hegemo- 
nía gestada desde los países centro del sistema mundo, permitió una transformación en las miradas y mecanismos de dominación y disciplina social en países periféricos.

Sin haber nacido como discurso de la globalización, el neoliberalismo terminó convertido en su expresión hegemónica, sinónimo de integración mundial, eficiencia económica, equidad social, progreso material y defensa del medio ambiente (Jiménez \& Novoa, 2014).

Ello permite considerar que con la globalización se desdibujan los limites, las fronteras estatales, pues éstas, a partir de lo que se conoce como financiarización, permite que problemas en torno al manejo de la política monetaria desde la especulación financiera, transforme la soberanía de los Estados: "la inversión es un motor importante para la productividad, dinamizadora del crecimiento económico" (Jiménez \& Novoa, 2014, p. 38-39).

La ecuación se ha invertido, los Estados ya no definen la política monetaria, ahora son los actores que ejercen dominio sobre ella los que definen las políticas estatales.

Las relaciones sociales se producen especialmente, ocurren en algún lugar, que va configurando las características necesarias para la producción y reproducción de esas relaciones. La destrucción creadora desprendida de la lógica capitalista, crea, ordena y funcionaliza paisajes, configuraciones territoriales que posteriormente, debido a su proceso de crisis, debe destruir, deshacer, reconfigurar espacialmente (Jiménez \& Novoa, 2014, p. 41).

La producción social del espacio está determinada por aquello que se conoce como capitalismo por desposesión, es decir, toda una serie de procesos y prácticas que mantienen la propiedad y el uso de derechos reales y patrimoniales (capacidad de adquirir y reorganizarse) en constante tránsito, de unas manos a otras, lo cual genera una afectación de poblaciones minoritarias frente a este tipo de dinámicas sociales; David Harvey (2012, p. 49) ha categorizado todas estas formas como acumulación primitiva. Aquí, el Estado juega un papel estructural, pues desde él se enuncian las formas legales que legi- 
timan estos procesos de dominación. La lógica global con la que se articula el capital mantiene una pluralidad de niveles (global, regional, nacional, sub-nacional), que a la hora de abordar los espacios se genera todo un proceso de desterritorialización/reterritorialización enunciado por agencias estatales y supraestatales, y ejercida desde el margen por toda una pluralidad de actores paraestatales o al margen de la ley. "Esta distorsión entre enunciación y práctica arroja una recomposición política y social del trabajo y de los grupos o sectores populares, busca la producción/consolidación de un nuevo lugar, un espacio diferenciado" (Jiménez \& Novoa, 2014, p. 41), es decir, bajo este prisma, las relaciones sociales solo son efecto de las relaciones capitalistas.

Las relaciones entre el espacio de vida y el espacio de producción se tornan difíciles de establecer, la relación entre lo íntimo, lo privado, lo público y lo común, manifiestan múltiples transformaciones. Los muros de las fábricas se transparentan, el mercado entra en la fábrica con la ayuda de nuevas tecnologías y la velocidad y fluidez del dinero, transformando las formas de producción, la gestión del trabajo, la distribución y el consumo (Jiménez \& Novoa, 2014, p. 31).

El problema de la estatalidad, así como la globalización, permite leer varios niveles de complejidad que se superponen entre sí. La biopolítica transforma las dinámicas sociales dentro del Estado, pues al introducirse en el campo de la economía quedan atravesadas y limitadas espacialmente, pero también frente al acceso de los recursos naturales, sin dejar de lado la forma para comprender la vida. Sin embargo, este ejercicio de dominación, en el que el Estado cede su lugar a la economía, no lo desplaza ni lo desaparece, no. El tránsito de este enroque configura una nueva razón de Estado donde la economía es la pieza principal para definir las relaciones sociales, no a partir de la coerción y la violencia (ésta no desaparece, solo se fortalece para reafirmar la autoridad), sino a partir de "la producción intensiva, flexible, móvil, con una alta composición orgánica de capital, una recomposición extensiva e intensiva del capital" (Jiménez \& Novoa, 2014). Estamos frente a nuevas tecnologías de gobierno donde se reconfiguran las relaciones sociales y la espacialidad. 
$[\mathrm{H}]$ ablar de "tecnologías políticas" significa preguntarse por la conducción eficaz de la conducta de otros para el logro de ciertos fines, por las estrategias que han de aplicarse razonadamente para lograr que las personas se comporten conforme a esos objetivos, y por el cálculo adecuado para elegir implementar esas estrategias (Castro, 2010, p.9).

Las tecnologías políticas que soportan el neoliberalismo generan un proceso de subjetivación de los individuos, y con ello, nuevos modos de existencia. La autorregulación de los sujetos, es decir, "lograr que los gobernados hagan coincidir sus propios deseos, esperanzas, decisiones, necesidades y estilos de vida con objetivos gubernamentales de ante mano" (Castro Gomez, 2010, p. 13), supone un efecto en cómo se están pensando los espacios, ya no desde su materialidad, sino desde su contingencia y virtualidad.

\section{La estigmatización de las protestas y los movimientos sociales en Colombia}

Una de las conquistas normativas que obtuvo la Constitución Política de 1991, hace 24 años, fue darle rango constitucional al derecho a protestar. Esto supuso un giro copernicano frente a la tradición que mantuvo el país en la segunda mitad del siglo XX donde, bajo una lógica schimmitiana (Schmitt, 1984), la excepción y el estado de sitio se postraron como regla en el ordenamiento jurídico colombiano (García, 2001, p. 319).

El artículo 37 de la Constitución categóricamente establece "[t]oda parte del pueblo puede reunirse y manifestarse pública y pacíficamente. Sólo la ley podrá establecer de manera expresa los casos en los cuales se podrá limitar el ejercicio de este derecho" (Republica de Colombia, 1991). Cuando la asamblea constituyente proyectó esta norma, pretendió superar las conquistas que habían adquirido la vida política nacional con el Estatuto de seguridad democrática del gobierno Turbay Ayala (1978-1982), el Estatuto para la defensa de la democracia del presidente Virgilio Barco (1986-1990) y el Estatuto para la defensa de la justicia de la administración de Cesar Gaviria (1990-1994) (Betancur, 2006), los cuales tuvieron como elemento común la suspensión 
total de derechos civiles e individuales, con ello, también se mantuvo la represión permanente de la protesta social (Uprimny \& Sánchez, 2010, p. 49).

El derecho a protestar, como garantía normativa o como derecho constitucional dentro de un ordenamiento jurídico, supone el reconocimiento, por parte del Estado, de aceptar el reclamo de derechos por vías no institucionales (Zaffaroni, 2009, p. 44). Sin embargo, por fuera de la rigidez con que se enuncian las normas, los derechos no dependen de su enunciación y su proyección en un texto, sino del reconocimiento de los actores que hacen ejercicio de los mismos, con ello, la protesta social y los movimientos sociales que han ejercido derecho sobre ella han quedado inmersos en una serie de estrategias estatales en las que se forjan toda una serie de técnicas de satanización sobre aquellos que proponen proyectos alternativos o hacen resistencia a las directrices con las que se administra la política en el país.

Desde inicios de la década de los 90 el calificativo de terrorista formó parte del discurso oficial que prendió justificar las detenciones arbitrarias, la desaparición y los asesinatos extrajudiciales. Poblaciones enteras que han defendido estrategias de resistencia han sido perseguidas y desplazadas de sus territorios, y parte de su dirigencia fue asesinada. El objetivo aquí no es solo eliminar al contrario, sino amedrentar, aleccionar, e impedir nuevas rearticulaciones de los movimientos y sujetos en resistencia. Aquí, el paramilitarismo ha sido completamente funcional a la política de Estado (Betancur, 2006).

En Colombia, la práctica legal ha reproducido una estigmatización de los movimientos sociales, con ello, se ha criminalizado por distintas vías la protesta social. El problema en torno a la protesta social en Colombia no es en cuanto a la enunciación de las normas que la reprochen penalmente, pues es evidente la cláusula constitucional, la protesta social es criminalizada a partir de la interpretación de la ley penal, es decir, los puntos de partida y las construcciones argumentativas que haga el operador judicial en torno a delitos que en principio protegen otra clase de derechos o situaciones. Con ello, vale decir que el problema en torno a la protesta en el contexto del conflicto armado, desde el punto de vista legal, ha dependido de la actitud del Estado frente a los grupos insurgentes, lo cual ha variado principalmente en dos tendencias: 
Por un lado, la búsqueda de su derrota a través de las vías militares y jurídicas y, por el otro, la búsqueda de acuerdos que permitan la superación del conflicto por la vía política. La situación de la criminalización de la protesta social en Colombia varía de conformidad con la tendencia imperante en cada momento histórico. Así en momentos en los que se privilegia la búsqueda de la derrota de los insurgentes, la criminalización de la protesta tiende a ser mayor (Uprimny \& Sánchez, 2010, p. 70).

La estigmatización de la protesta social en ese sentido, no es más que el efecto de la política estatal frente al conflicto, lo cual desdibuja del panorama toda posibilidad de expresiones alternativas o reclamos de derechos. La invención de enemigos por parte del Estado, mantiene una lógica binaria en la que cualquier posición será calificada a favor o en contra, imposibilitando el pensamiento crítico, suprimiendo aquella máxima en la que "[e]l mundo que queremos es uno donde quepan muchos mundos" (Marcos \& Le Bot, 2011). Son tres las razones que explican esta dinámica (Uprimny \& Sánchez, 2010, p. 69):

1. Los grupos guerrilleros e insurgentes son estructuras políticomilitares interesadas en tener presencia en los movimientos sociales;

2. la sospecha de la participación de estos grupos, estigmatiza a los activistas sociales como militantes, colaboradores o simpatizantes de la guerrilla;

3. eventualmente, a partir de beneficios judiciales por información, se proporciona información falsa sobre la cual se construyen montajes judiciales contra activistas sociales.

\section{CONCLUSIONES}

Los marcos explicativos de los movimientos sociales en Colombia, si bien son plurales en la forma en como pretenden describir y comprender sus dinámicas, guardan relación en ciertos aspectos metodológicos, permitiendo identificar cuándo un movimiento es sólido en sus acciones colectivas. Así mismo, la característica común de esta analítica de los movimientos converge en que sus motivaciones se consolidan a 
partir de procesos de transformación social. Los efectos políticos de los movimientos sociales no se limitan a ejercer presión política con la institucionalidad, sino que se concentra en espacios alternativos de deliberación que permiten resignificar los presupuestos de la democracia. Sin embargo, la acción colectiva es neutralizada en el momento que la institucionalidad se vuelve difusa, atendiendo a criterios externos a las funciones de bienestar y garantía que el Estado supone.

A partir del prisma de la gubernamentalidad se transforman las funciones del Estado moderno, y con ello, la noción de soberanía. Si bien el Estado mantiene cierto protagonismo el campo de lo público y la política, ahora con las dinámicas de la(s) globalización(es) se torna en un actor más a la hora de enunciar directrices que impacten en la población: empresas multinacionales, instituciones jurídicas supraestatales, las ONG, organizaciones sociales y políticas, etc.; "la reconfiguración geopolítica nacional e internacional tiene que contar con la presencia y acción estatal a nivel interno de los países, como el ámbito transnacional, aunque sea un Estado ahuecado" (Jiménez \& Novoa, 2014, p. 49).

En ese sentido, si bien es importante tener un marco normativo lleno de garantías que impida la criminalización de la protesta social y de movimientos sociales, ya sea apelando a la libertad de opinión, el libre desarrollo de la personalidad o la libertad de reunión, es más significativo tener argumentos frente a como es criminalizada la protesta social desde las formas jurídicas establecidas, pues el lenguaje del derecho, con su sacralidad y neutralidad, si bien constituye una herramienta que legitima una política de Estado represiva o captadora de garantías individuales, también en él existen las posibilidades y los puntos de fuga para construir un discurso que no solo le dé las garantías a los más débiles (Ferrajoli, 2004), sino que con él puedan darle la voz a los que histórica y sistemáticamente han estado sin voz. Con esto, el derecho penal y sus actores: fiscales, jueces, magistrados, etc. deben asumir una posición diferente del guardián de la ley de aquel relato de Kafka (Kafka, 2005), cederle el secreto de la ley sin prevención alguna, comprendiendo que al hacerlo éste ya no es necesario. 
MOVIMIENTOS SOCIALES, NUEVA RAZÓN DE ESTADO Y LA

ESTIGMATIZACIÓN DE LA PROTESTA SOCIAL EN COLOMBIA

\section{REFERENCIAS}

Betancur, M. (2006). Del Estatuto de Seguridad al estado comunitario: Veinticinco años de criminalización de la protesta social en Colombia. OSAL, Observatorio Social de América Latina, 179-224.

Castro, S. (2010). Historia de la gubernamentalidad: razón de estado, liberalismo y neoliberalismo en Michel Foucault. Bogotá: Siglo del Hombre Editores.

Delgado, R. (2005). Análisis de los marcos de acción colectiva en organizaciones sociales de mujeres, jóvenes y trabajadores. Manizales: Universidad de Manizales.

Ferrajoli, L. (2004). Derechos y garantías: la ley del más débil. Madrid: Trotta.

Foucault, M. (2001). Defender la sociedad. Curso en el College de France (1975-1976). Buenos Aires: Fondo de Cultura Económica.

García, M. (2001). Constitucionalismo Perverso. Normalidad y anormalidad constitucional en Colombia: 1957-1997. En B. d. Santos, \& M. García, El caleidoscopio de las justicias en Colombia. Bogotá: Universidad de los Andes, Siglo del Hombre. p. 317-370.

Harvey, D. (2012). Ciudades rebeldes. Del derecho a la ciudad a la revolución urbana. Madrid: Akal.

Jiménez, C., \& Novoa, E. (2014). Producción social del espacio: El capital y sus luchas sociales en la disputa territorial. Bogotá: Ediciones desde abajo.

Kafka, F. (2005). Ante la ley. Escritos publicados en vida. Bogotá: DeBolsillo.

Marcos, S., \& Le Bot, Y. (Febrero de 2011). Biblioteca Social d'Olot. Obtenido de El Sueño Zapatista. Disponible en: http://www.bsolot.info/wp-content/ uploads/2011/02/Marcos_Subcomandante_Bot_Yvon-El_sue\%C3\%B1o_ zapatista.pdf

Melucci, A. (1994). ¿Qué hay de nuevo en los movimientos sociales? En E. Laraña, Los nuevos movimientos sociales: De la ideología a la identidad (p. 118-145). Madrid: CIS.

Melucci, A. (1996). Challenging Codes: Collective Action in the Information Age. Cambridge: Cambridge University Press. 
Melucci, A. (1999). Acción colectiva, vida cotidiana y democracia. México D.F.: El Colegio de México.

Neveu, E. (2000). Sociología de los movimientos sociales. Quito: Abya Yala.

Ragin, C. (2007). La construcción de la investigación social. Introducción a los métodos y su diversidad. Bogotá: Siglo del hombre editores.

República de Colombia. Consejo Superior de la Judicatura (2010). Constitución Política de Colombia de 1991. Bogotá: Imprenta Nacional de Colombia.

Schmitt, C. (1984). El Concepto de lo "político"; Teoría del partisano: notas complementarias al concepto de lo "político". Folios: Buenos Aires.

Uprimny, R., \& Sánchez, L. (2010). Derecho Penal y Protesta Social. En E. A. Bertoni, ¿Es legítima la criminalización de la protesta social?: Derecho Penal y Libertad de Expresión en América Latina. Buenos Aires: Universidad de Palermo. p. 47-74.

Zaffaroni, E. (2009). Derecho Penal y Protesta Social. En E. Zaffaroni, Hacia dónde va el poder punitivo. (33-52). Medellín: Universidad de Medellín. 